\begin{tabular}{|l|l|l|l|}
\hline Eiszeitalter u. Gegenwart & $\mathbf{2 7}$ & $\begin{array}{l}18-25 \\
3 \mathrm{Abb} .\end{array}$ & Öhringen/Württ. 1976 \\
\hline
\end{tabular}

\title{
Ergebnisse paläomagnetischer Untersuchungen an quartären Sedimenten des Rhein-Main-Gebiets
}

\author{
Arno Semmel \& Kurt Fromm*)
} Stratigraphic classification, paleomagnetism, loess, pleistocene fluvial sediments,
Federal Republic of Germany, Rhine-Main-Region

\begin{abstract}
Kurzfassung: Als erste Ergebnisse der derzeit laufenden paläomagnetischen Untersuchungen im Rhein-Main-Gebiet werden die Befunde von einigen stratigraphisch bedeutenden Profilen mitgeteilt. Während die Remanenzwerte aus altpleistozänen Tonen der Kelsterbacher Terrasse mit einer Ausnahme revers sind, konnte im Autobahneinschnitt Abenheim (NW Worms) und im Lößprofil der Ziegeleigrube Bad Soden am Taunus der Übergang von reverser zu normaler Magnetisierung gefunden werden. Die Grenze zwischen Matuyama- und Brunhes-Epoche liegt im Profil Bad Soden unter dem 6., das Jaramillo-Event unter dem 7. fossilen $\mathrm{B}_{\mathrm{t}}$-Horizont.
\end{abstract}

[Results of Paleomagnetic Investigations on Quaternary Sediments in the Rhine-Main-Region]

A bstract: First results of paleomagnetic investigations presently being carried out in the Rhine-Maine-Region on some stratigraphically important profiles are reported. The remanent magnetization of old-pleistocene fluvial clay sediments in the "Kelsterbacher Terrasse" is, with one exception, reverse, whereas in the cutting of the motorway Abenheim (northwest of Worms) and in the loess profile of the brickyard at Bad Soden am Taunus the transition from reverse to natural remanent magnetization could be found. The boundary between the Maturama and BRUnHes epoch in the profile Bad Soden am Taunus lies underneath the sixth, the Jaramillo Event under the seventh fossil B-horizon of a parabrown earth (lessivé).

\section{Einleitung}

In jüngster Zeit hat sich die Paläomagnetik bei der stratigraphischen Einordnung von Quartärprofilen häufig als wichtiges Hilfsmittel erwiesen. Von besonderer Bedeutung war die Idee Kunlas, die Paläomagnetik auf das Lößprofil am Roten Berg bei Brünn anzuwenden (Bucha et al. 1969). Seitdem sind viele Lößprofile paläomagnetisch untersucht worden (z. B. BondArschuk et al. 1976; BRUNNACKer et al. 1976; FINK et al. 1976), denn der Löß ist besser als jedes andere terrestrische Sediment - wegen seiner oft über lange Zeitabschnitte hinweg kontinuierlichen Ablagerung - geeignet, Veränderungen des erdmagnetischen Feldes im Pleistozän wiederzugeben. Er ist hierin vor allem den Sanden und Tonen überlegen, in denen paläomagnetische Messungen wegen der groben Körnung der Sande bzw. der geringen Mächtigkeit vieler Tonlagen oft nicht zu brauchbaren Ergebnissen führen. Dennoch erweist sich die Paläomagnetik auch bei solchen Ablagerungen in Verbindung mit anderen stratigraphischen Methoden als wesentliche Hilfe für die Alterseinstufung von aquatischen Ablagerungen, wie z. B. die Untersuchungen von MonTrRans (1971), WIEGANK (1975) und anderen Autoren zeigen.

Im Rhein-Main-Gebiet (Abb. 1), also im nördlichsten Teil der oberrheinischen Tiefebene, sind in größerer Mächtigkeit quartäre Sedimente abgelagert worden, die erwarten ließen, daß in ihnen markante paläomagnetische Leithorizonte, z. B. die Grenze der Matuyama- zur Brunhes-Epoche, zu finden sind. Hierzu gehören einerseits die durch

\footnotetext{
*) Anschriften der Verfasser: Prof. Dr. S e m m e 1, Geographisches Institut der Johann-Wolfgang-Goethe-Universität, Senckenberganlage 36, 6000 Frankfurt/Main; K. F r o m m , Dipl.-Geophys., Niedersächs. Landesamt für Bodenforschung, Stilleweg 2, Alfred-Bentz-Haus, 3000 Hannover 51 .
} 
mehrere Tonlagen gegliederten altpleistozänen $\left(t_{(1)}\right)$-Kiese des Mains und Rheins (SemmeL 1974), andererseits aber vor allem mehrere Lößprofile, die aufgrund ihrer Lage und der in ihnen enthaltenen zahlreichen fossilen $\mathrm{B}_{\mathrm{t}}$-Horizonte als zumindest in ihren unteren Teilen altpleistozän einzustufen waren.

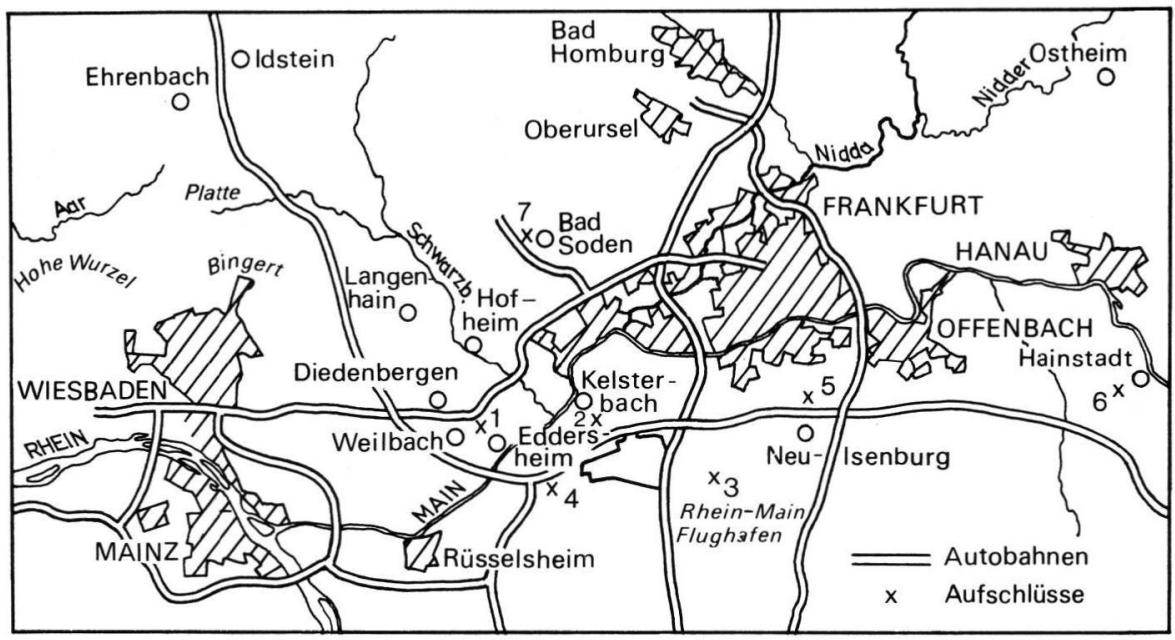

Abb. 1: Lage der untersuchten Profile.

$1=$ Kiesgrube Jak. Müller bei Weilbach; 2 = Kiesgrube am Umspannwerk Kelsterbach; $3=$ Kiesgrube am Gehspitz; $4=$ Kiesgrube Mitteldorf am BAB-Dreieck Mönchhof; $5=$ Kiesgrube Fr. Bauer bei Neu-Isenburg; 6 = Ziegeleigrube Hainstadt; $7=$ Ziegeleigrube Bad Soden.

Durch die freundliche Unterstützung von Herrn Kollegen Minato, Sapporo, bot sich bereits 1972 die Gelegenheit, jeweils eine Probenserie aus fluvialen Tonen und aus Lößlehmen durch Herrn Dr. Fujiwara vom Department of Geology der Universität Sapporo paläomagnetisch untersuchen zu lassen. Während die Tone keine verwertbaren Daten lieferten, zeigte der Lößlehm eindeutig reverse Magnetisierung (SEMmEL 1973: 296). Es handelte sich dabei um Proben, die aus dem unteren Teil des Lößprofils der ehemaligen Ziegeleigrube Bad Soden am Taunus stammten. Dieses Lößprofil enthält mit insgesamt acht fossilen $\mathrm{B}_{\mathrm{t}}$-Horizonten und dem basalen fossilen Pseudogley eine besonders hohe Zahl intensiv ausgeprägter Paläoböden (SEMMEL 1967). Im Rahmen paläomagnetischer Untersuchungen des Niedersächsischen Landesamtes für Bodenforschung an quartären Sedimenten begann K. Fromm 1972 mit einer eingehenden Probennahme sowohl im Profil Bad Soden als auch in einigen Kiesgruben des Untermaingebietes, in denen altpleistozäne Tonlagen aufgeschlossen waren. Gleichzeitig, jedoch völlig unabhängig, liefen von BRUNNACKer geleitete Arbeiten, die u. a. das Profil Bad Soden betrafen und über die bereits berichtet wurde (Koči et al. 1973; BoENIGK et al. 1974; BrunNACKER et al. 1976). Die letztgenannte Publikation war uns zum Zeitpunkt des Vortrages (SemmeL) auf der DEUQUATagung in Hamburg 1976 noch nicht bekannt. Die nachstehend mitgeteilten Befunde sind das Ergebnis des ersten Teils unserer Untersuchungen. Die Auswertung des gesamten Probenmaterials, insbesondere die der Proben aus Würmlößprofilen, ist noch nicht abgeschlossen. K. Fromm wird außerdem über methodische Fragen an anderer Stelle berichten. Ein Protokoll über die Untersuchungen befindet sich im Archiv des Niedersächsischen Landesamtes für Bodenforschung, Hannover (Nr. 76256). 


\section{Probennahme; Mittelung und Beurteilung der Remanenzrichtungen}

Zur Prüfung des Aussagewertes der remanenten Magnetisierung wurden zunächst aus einigen Horizonten mehrere Proben gestochen. Ein Stechrohr mit quadratischem Querschnitt ( $3 \mathrm{~cm}$ Kantenlänge), das sich in weichen Tonlagen bewährt hatte, erwies sich im Löß als weniger geeignet. Es wurde daher so modifiziert, daß die Probe im Stechrohr in eine Plastikhülle gleitet, in der sie transportiert und konserviert wird.

Die ersten Ergebnisse zeigten so stark streuende Richtungen, daß vor allem für die unteren Schichten des Profils Bad Soden keine Aussage möglich war (vgl. auch BRUNNACKER et al. 1976: 364). Das verbesserte Stechrohr ermöglichte dann aber mit vertretbarem Zeitaufwand hinreichend viele Proben (ca. 10) aus jedem der meist 10 bis $15 \mathrm{~cm}$ übereinanderliegenden Stechzeilen zu gewinnen, um mittlere Richtungen zu errechnen, die schließlich doch ein zufriedenstellendes Resultat lieferten. In Abb. 2 ist zu jedem Mittelwert die Anzahl der Meßwerte angegeben. Bei der Beurteilung der Daten ist zu beachten, daß nur e in e physikalische Größe, nämlich die räumliche Richtung des paläomagnetischen Remanenzvektors, betrachtet wird. Eine sehr gute Darstellung räumlicher Richtungen bietet die stereographische Polarprojektion, wie sie in Abb. 3 benutzt wird. Um die Abfolge von Remanenzrichtungen in einem Quartärprofil darzustellen, muß man allerdings auf die weniger anschauliche Zerlegung der Richtung in die gekoppelten Winkel Inklination und Deklination ausweichen. Die Betrachtung einer dieser Darstellungskomponenten - insbesondere der Deklination - losgelöst von der anderen, führt leicht zu Fehlurteilen, denn schon geringe Orientierungs- oder Meßungenauigkeiten können bei steiler Inklination die Deklination um beliebige Werte ändern. In Sedimenten können die Form der magnetischen Minerale und die Ablagerungsbedingungen neben der magnetischen Orientierung der Teilchen einen systematischen Einfluß auf den Remanenzvektor haben, so daß auch nach Eliminieren viskoser Remanenzanteile nicht notwendig die Richtung des paläomagnetischen Feldes wiedergegeben wird. Ein solcher Einfluß kann in den unteren Schichten unseres Profils eine Rolle spielen, wo die fast horizontale Südrichtung der Remanenz nach Abwägen verschiedener Indizien ${ }^{1}$ ) nur der Matuyama-Epoche zugeordnet werden kann.

\section{Zur Paläomagnetik des Lößprofils Bad Soden}

Es wurde schon betont, daß in dem Lößprofil der ehemaligen Ziegeleigrube Bad Soden am Taunus acht fossile $B_{t}$-Horizontreste und ein fossiler Pseudogley im Löß bzw. Lößlehm aufgeschlossen sind. Darunter liegt ein altpleistozäner Schotterkörper über weißen pliozänen Tonen mit Linsen von kantigen Quarzen. Eine Gesamtskizze des Aufschlusses ist bei SeMmel (1974: 196) zu finden. Die für die paläomagnetischen Befunde wichtigen Horizonte sind auf Abb. 2 wiedergegeben. Auf dieser Abbildung wird der Teil der Grubenwand dargestellt, der heute noch am besten zugänglich ist und zugleich die wesentlichsten Straten, die paläomagnetisch bedeutsam sind, enthält. Etwa in diesem Bereich wurden wahrscheinlich auch die Proben von BrunNacker et al. (1976: 364) entnommen.

Die paläomagnetischen Richtungen liegen im Löß unter dem Lohner Boden des Mittelwürms (SснӧNHALs et al. 1964) bis zum Löß, aus dem der fünfte (von oben gezählt) fossile $\mathrm{B}_{\mathrm{t}}$-Horizontrest hervorgegangen ist, $\mathrm{n}$ or $\mathrm{m}$ a l. Innerhalb des Lösses (es handelt sich um entkalkten Löß, also um Lößlehm) unter und innerhalb des sechsten fossilen $B_{t}$-Horizontrestes liegt die Umkehr in die reverse Magnetisierung. Die Reversrichtung bleibt in diesem und dem tieferen Profilteil konstant. Nur unter dem siebenten fossilen $B_{t}-$ Rest

1) Indizien sind die generelle Übereinstimmung mit der Alterseinstufung der Schichten zusammen mit dem markanten Wechsel von einer Generalrichtung zu einer deutlich anderen, ferner das Abmagnetisierungsverhalten auch der Remanenzbeträge in einzelnen Fällen sowie die vollendete Gegenrichtung beim (Jaramillo-) Event. 


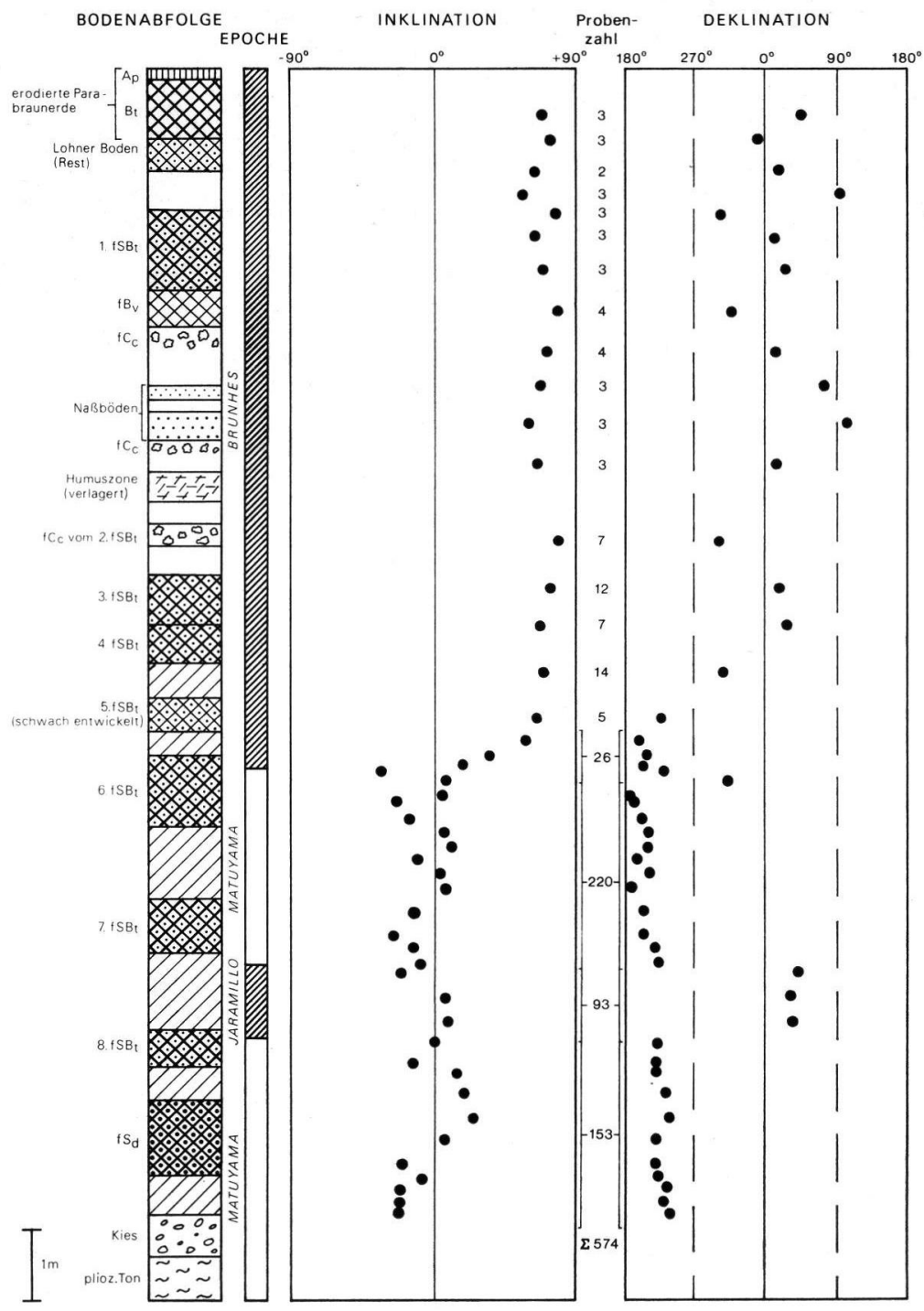

Abb. 2: Bodenabfolge und paläomagnetische Remanenzrichtungen (Mittelwerte) in der ehemaligen Ziegeleigrube Bad Soden a. Ts.

Kalkhaltiger Löß ist weiß, kalkfreier Löß (außerhalb der eigentlichen Böden) schräg schraffiert dargestellt. Die Punkte geben die Lage der Stechzeilen an, aus denen die Proben entnommen wurden. Der skizzierte Profilausschnitt liegt in der Mitte der Gruben-Nordwand, die parallel zum $\mathrm{Zu}-$ bringer zur Autobahn Ffm.-Höchst/Königstein verläuft.

$\mathrm{fSB}_{\mathrm{t}}$ bedeutet: fossiler pseudovergleyter Tonanreicherungshorizont. Für den 1. $\mathrm{fSB}_{\mathrm{t}}$ kann mit $\mathrm{Si}$ cherheit angenommen werden, daß er der letzten Warmzeit (Riß/Würm) angehört. Für den 2. fSB ist die Zugehörigkeit zur vorletzten Warmzeit sehr wahrscheinlich. Die stratigraphische Stellung der übrigen $\mathrm{fSB}_{\mathrm{t}}$-Horizonte ist unklar. Der Grenze MatuYama-Brunhes wird gegenwärtig allgemein ein Alter von ca. 700000 Jahren zuerkannt. Das Jaramillo-Event soll zwischen 880000 und 960000 a.B.P. liegen (vgl. dazu u. a. Glass et al. 1967). Unter der - nicht sicheren - Voraussetzung, daß in den Abtragungsdiskordanzen dieses Profils nicht noch weitere $\mathrm{B}_{\mathrm{t}}$-Horizonte gelegen haben, hat es innerhalb der letzten 700000 Jahre im Pleistozän sechs wärmere Abschnitte gegeben, in denen die Entwicklung von Böden mit $\mathrm{B}_{\mathrm{t}}$-Horizonten möglich war. Zumindest für den sehr schwach entwickelten $5 . \mathrm{fSB}_{\mathrm{t}}$ ist fraglich, ob es sich dabei um eine echte Warmzeit gehandelt hat. 


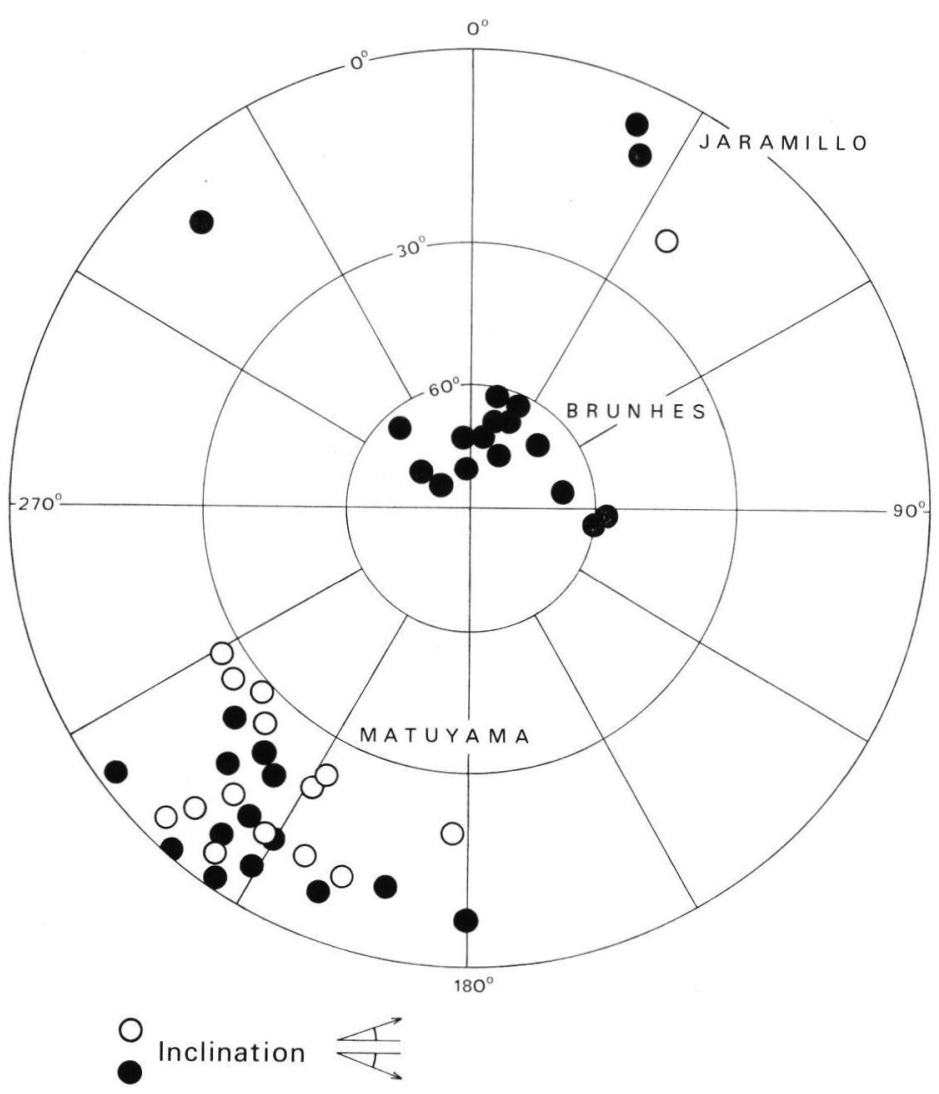

Abb. 3: Stereographische Projektion der Probenentnahmestellen.

Die stereographische Projektion zeigt deutlich die Verschiedenheit der Remanenzrichtung in der BRunhes-Epoche und in der Matuyama-Epoche mit ihrer Umkehr im Jaramillo-Event.

schlägt die Richtung abrupt um $180^{\circ}$ in die als normal anzusprechende Magnetisierung um. Der Lößlehm des achten fossilen $B_{t}$-Restes zeigt aber bereits wieder reverse Magnetisierung. Diese bleibt mit guter Konstanz bis dicht über dem liegenden Kies erhalten (Abb. 2).

Unter Beachtung der derzeit in der Regel angewandten stratigraphischen Paläomagnetik-Skala (GLAss et al. 1967; Cox 1969) kann davon ausgegangen werden, daß im Profil Bad Soden die Lößsedimentation vor mehr als einer Million Jahren mit reverser Magnetisierung begann. Das Liegende bildet ein Schotterkörper, der zu den altpleistozänen Taunusrand-Terrassen gehört (SEmmel 1969: 52). Aufgrund der Terrassenabfolge ist davon auszugehen, daß dieser Schotterkörper mit Sicherheit jünger als die Tegelen-Warmzeit und älter als der Cromer-Komplex ist (vgl. dazu S. 24). Der tiefste Löß muß danach jünger als die älteste Warmzeit (Tegelen) sein. Das erste Vorkommen normaler Magnetisierung im Löß unter dem siebenten fossilen $B_{t}$-Rest kann demnach nicht dem Gilsa-Event, das Montfrans (1971) dem unteren Tegelen gleichsetzt, entsprechen, sondern am ehesten dem Jaramillo-Event, das nach Montfans in den oberen Teil der Waal-Warmzeit zu stellen ist. Jedoch sind auch Sedimente der nach oben anschließenden Menap-Kaltzeit noch normal magnetisiert (ZAGwIJN et al. 1971: 54). Dabei bleibt zu berücksichtigen, daß der 
Löß keine warmzeitliche Bildung ist, also nach der Waal-Warmzeit abgelagert worden sein dürfte. Vorher entstanden mit dem basalen Pseudogley und dem achten fossilen $\mathrm{B}_{\mathrm{t}}{ }^{-}$ Rest zwei sogenannte „Warmzeit-Böden“, obwohl allenfalls eine - paläobotanisch nachgewiesene - Warmzeit, nämlich die Waal-Warmzeit, sich auswirken konnte. Hier zeigt sich die Problematik, die bei der Parallelisierung von methodisch verschiedenen stratigraphischen Einstufungen oft entsteht. Die Frage wäre gelöst, wenn es im Pleistozän 1. mehr Parabraunerden als Warmzeiten gegeben hätte, 2. mehr Warmzeiten als bisher paläobotanisch nachgewiesen existierten oder 3. noch andere paläomagnetische Events im Altpleistozän vorkommen. Unseres Erachtens kann indessen im Augenblick keine der angeführten Möglichkeiten mit Sicherheit ausgeschlossen werden.

Im Profil Bad Soden stellt sich im Lößlehm des sechsten fossilen $B_{t}$-Restes allmählich die normale Magnetisierung ein. Dieser Wechsel kann nur als die Grenze der Matuyamazur BRUNHES-Epoche gedeutet werden. In dem darüber folgenden Löß, der oberhalb des dritten fossilen $B_{t}$-Restes mit Ausnahme der intensiveren Böden kalkhaltig ist, dominiert die normale Magnetisierung. Inwieweit in diesem stratigraphischen Bereich noch Events vorkommen können, sollen derzeit noch nicht abgeschlossene Untersuchungen an mächtigeren jüngeren Lössen im Rhein-Main-Gebiet ergeben.

Vergleicht man die hier erörterten Befunde mit den Ergebnissen von BRUNNACKER et al. (1976), so ergeben sich doch recht gute Übereinstimmungen. Das Jaramillo-Event wird z. B. von Brunnacker et al. im Bereich des Paläobodens 7 vermutet. Aufgrund unserer Ergebnisse läßt sich die Formulierung „mit großer Wahrscheinlichkeit“ vertreten, denn im Unterschied zu BrunNaCKer et al. ist - wohl aufgrund anderer Meßmethodik (vgl. S. 20) - die Streuung der Ergebnisse in unserem Profil nicht so stark.

Auch hinsichtlich der Matuyama-Brunhes-Grenze ergibt sich Übereinstimmung insofern, als diese Grenze im Bereich des Lösses liegt, aus dem der sechste fossile $B_{t}$-Rest hervorging. Abweichend von BRUNNACKER et al. (1976: 364-365) liegt allem Anschein nach aber in unserem Entnahmebereich keine einschneidende Diskordanz. Hier kommt zusätzlich ein Interpretationsunterschied zum Tragen, auf den BRUNNACKER et al. hinweisen: Der Paläoboden 6 im Sinne Brunnackers et al. ist nach der Auffassung von Semmel (1974: 196), ebenso wie ältere und auch noch iüngere fossile Böden, durch einen kleinen Grabeneinbruch gestört, der bei der Zählung der Böden leicht zu Abweichungen führen kann (vgl. auch RAZI RAD 1976: 87).

Mit dieser Feststellung soll nicht behauptet werden, daß es im Lößprofil Bad Soden keine Diskordanzen gibt - einige von ihnen sind auf Abb. 2 dargestellt - nur hinsichtlich der wichtigsten paläomagnetischen Straten scheinen uns in Bad Soden die zur Zeit als stratigraphisch besonders bedeutsam angesehenen oberhalb des Gilsa-Events in einer Deutlichkeit vorzuliegen, wie sie bisher aus terrestrischen Sedimenten im Bereich der Bundesrepublik nicht bekannt wurden. Das Profil Bad Soden ist außerdem das einzige Profil im westlichen Mitteleuropa, das eine solche paläomagnetische Entwicklung ausschließlich im Löß bzw. Lößlehm aufweist.

Auf die Parallelisierungsmöglichkeiten mit den Profilen Kärlich und anderen ist von BrunNaCKer et al. schon eingegangen worden. Aus unserer Sicht ergibt sich dazu keine gravierend abweichende Deutung. Bei einem Vergleich mit dem Roten Berg bei Brünn fällt auf, daß bis zum Jaramillo-Event in beiden Aufschlüssen sieben sogenannte Warmzeit-Böden im hangenden Löß ausgebildet sind. Hier ist allerdings die Einstufung dieses Events als Inversion an der Grenze Matuyama/Brunhes durch Bucha et al. (1969: 127) im Profil Roter Berg zu beachten (vgl. neuerdings Bucha et al. 1975: 129). Im Bereich von ca. 700000 a.B.P., in dem die Grenze Matuyama/Brunhes üblicherweise angenommen wird (z. B. Cox 1969), liegt genau wie in Bad Soden auch am Roten Berg der sechste fossile „Warmzeit-Boden“ (PK VIII der tschechischen Lößforscher). In Bad Soden 
gehört aber hierzu im hangenden Löß auch der fünfte fossile $B_{t}$-Rest, der sehr schwach ausgebildet ist und u. E. sehr wahrscheinlich kein echter Warmzeitboden ist. Inwieweit das für die anderen fossilen $B_{t}$-Horizonte zutrifft, bleibt wohl weiterer Forschung vorbehalten. Im gegenwärtigen Zeitpunkt scheint eine Einstufung intensiver Löß-Parabraunerden oder ihrer Reste als jeweils interglaziale Bildung zwar nicht unberechtigt, jedoch auch noch nicht bewiesen. In diesem Zusammenhang sei auf wiederholte frühere Stellungnahmen verwiesen (u. a. Semmel 1967: 241; 1968: 10; 1974: 30; 1977: 78).

\section{Paläomagnetische Messungen in aquatischen pleistozänen Sedimenten des Rhein-Main-Gebietes}

Im Rhein-Main-Gebiet gibt es mit den Mosbacher Sanden und der Kelsterbacher Terrasse altpleistozäne Sedimente, die relativ große Mächtigkeit erreichen und teilweise sehr fossilreich sind. Es bot sich an, die bisher vorliegenden stratigraphischen Betunde (vgl. dazu Semmel 1974) durch paläomagnetische Messungen zu überprüfen bzw. zu ergänzen. KočI et al. (1973) teilten bereits die Ergebnisse entsprechender Untersuchungen aus den Mosbacher Sanden mit, deren stratigraphische Stellung zuletzt ausführlich von BRÜNING (1974) erörtert wurde. Brauchbare Ergebnisse sind bei unseren Arbeiten nur aus feinkörnigen Ablagerungen (Schluffe und Tone) gewonnen worden. Es wurden Bildungen des jüngsten Pliozäns und der ältesten pleistozänen Warmzeiten in den Aufschlüssen Kiesgrube Jakob Müller östlich Weilbach, Kiesgrube am Umspannwerk Kelsterbach, Kiesgrube am Gehspitz westlich Buchschlag, Kiesgrube Mitteldorf am Autobahndreieck Mönchhof, Kiesgrube Fr. Bauer nördlich Neu-Isenburg und Ziegeleigrube Hainstadt bei Hanau untersucht. Die einzelnen Horizonte sind aufgrund pollenanalytischer Untersuchungen (v. D. BRELIE 1974; außerdem noch nicht publizierte Ergebnisse von Borger) als dem Pliozän, der Tegelen-, der Waal- und der Cromer-I-Warmzeit angehörende Ablagerungen eingestuft worden. In keinem Fall handelt es sich allerdings um Horizonte, die ein gesamtes Interglazial umfassen. Es überrascht deshalb nicht, daß mit Ausnahme der oberen Tonlage in der Ziegeleigrube Hainstadt nur reverse Magnetisierung ermittelt wurde. Die normale Lagerung in Hainstadt widerspricht nicht der pollenanalytischen $\mathrm{Zu}-$ ordnung dieses Tons in die Tegelen- oder Waal-Warmzeit. Eine paläobotanisch belegte Ablagerung der Cromer-II-Warmzeit, deren Sedimente nach ZAGWIJN et al. (1971) normal magnetisiert sind, ist gegenwärtig im Rhein-Main-Gebiet nicht aufgeschlossen.

Den Wechsel von reverser zu normaler Polarität zeigen dagegen Ablagerungen, die beim Bau der Autobahn südlich Abenheim, vordwestlich Worms, aufgeschlossen waren. Herr Dr. Beck, Mainz, der uns auf diesen Aufschluß hinwies, wird an anderer Stelle ausführlicher über die Lagerungsverhältnisse berichten. Für die Stratigraphie und die paläomagnetischen Messungen war dieser Aufschluß insofern besonders interessant, als unter einer Lößdecke ca. $10 \mathrm{~m}$ mächtige graue Mosbacher Sande lagen („Graues Mosbach“, i. S. von BRüNING 1974: 64; „Mittleres Mosbach“, i. S. der älteren Literatur). Darunter folgten rötliche lehmige Sedimente (1-2 m mächtig), dann ca. $8 \mathrm{~m}$ mächtige gelblichbraune Hochflutlehme und Lösse (teilweise umgelagert) mit einem $B_{t}-$ Rest und einer Humuszone. Diese gelblichbraunen Sedimente waren durch einen rötlichbraunen Kies von ca. $1 \mathrm{~m}$ Stärke unterteilt. Das Liegende bildete wieder ein rötlichbrauner Kies von ca. $4 \mathrm{~m}$ Mächtigkeit, unter dem pliozäner Sand lag. Die in den Sedimenten zwischen pliozänem Sand und den Mosbacher Sanden entnommene Probenserie zeigt im unteren Teil, nämlich in lehmigen Schmitzen des rötlichbraunen Kieses, reverse Magnetisierung. Die hangenden gelbbraunen Lehme sind ausnahmslos normal polarisiert, die unmittelbar unter den Mosbacher Sanden liegenden rötlichen Lehme ebenfalls. Die Entscheidung, ob die Umkehr der Polarität in

diesem Profil der Grenze Matuyama/Brunhes entspricht oder dem Übergang zum Jaramillo-Event, ist schwierig. Im Gegensatz zu Bad $\mathbf{S} \sim$ - len tritt hier die negative Inklination 
deutlich hervor. Das kann jedoch auch sedimentbeuingt sein. Ein älteres Event als Jaramillo sollte nicht in Frage kommen, denn der liegende, revers magnetisierte Kies besitzt schon typisch pleistozäne Fazies.

Weiteren Aufschluß in dieser Frage werden vermutlich die Untersuchungen von ähnlichen Ablagerüingen in anderen Teilen Rheinhessens ergeben, die gegenwärtig gemeinsam mit W. Plass ausgeführt werden. Das Interesse gilt vor allem den in diesen ältestpleistozänen Sedimenten vorkommenden roten Böden, die u. a. den von Fink (1976: 102-103) aus Niederösterreich beschriebenen gleichen. Bisher waren solche Böden im westlichen Mitteleuropa noch nicht bekannt. Sie fehlen z. B. auch in Bad Soden (vgl. zu dieser Frage Semmel 1977: 78).

\section{Schriftenverzeichnis}

Boenigk, W., Heye, D., Schirmer, W. \& Brunnacker, K. (1974): Paläomagnetische Messungen an vielgliedrigen Quartär-Profilen (Kärlich/Mittelrhein und Bad Soden i. Taunus). - Mainzer naturwiss. Arch., 12: 159-168; Mainz.

BondarschuK, V. G. (Chef-Herausgeber) (1976): Führer für das 8. Internationale Löß-Symposium. -72 S.; Kiew.

BreLIe, G. vON DER (1974): Pollenanalytische Untersuchungen an warmzeitlichen Sedimenten in den Terrassen des Untermain-Gebietes. - Rhein-Main. Forsch., 78: 83-99; Frankfurt a. Main.

BRÜNING, H. (1974): Das Quartärprofil im Dyckerhoff-Steinbruch Wiesbaden/Hessen. - RheinMain. Forsch., 78: 83-99; Frankfurt a. Main.

Brunnacker, K., Boenigk, W., Koči, A. \& Tillmanns, W. (1976): Die Matuyama/Brunhes-Grenze am Rhein und an der Donau. - N. Jb. Geol. Paläont. Abh., 151: 358-378; Stuttgart.

Bucha, V., Horáčex, J., Koči, A. \& KuKLA, J. (1969): Paläomagnetische Messungen in Lössen. In: Periglazialzone, Löß und Paläolithikum in der Tschechoslowakei: 123-131; Brünn.

Bucha, V., Horáčex, J., ŠIBrava, V. \& LožEK, V. (1975): Paleomagnetic Correlations of Pleistocene Sediments of Glaciations in the Northern Hemisphere, Rep. 2: 9-36; Praha.

Cox, A. (1969): Geomagnetic Reversals. - Sci., 163: 237-245; Washington, DC.

Fink. J. (Schriftleiter) (1976): Exkursion durch den österreichischen Teil des nördlichen Alpenvorlandes und den Donauraum zwischen Krems und der Wiener Pforte. - Mitt. Komm. Quartärforsch. österr. Akad. Wiss., 1: 113 S.; Wien.

Glass, B., Ericson, D. B., Heezen, B. C., Opdike, N. D. \& Glass, J. A. (1967): Geomagnetic Reversals and Pleistocene Chronology. - Nature, 216: 437-442; London.

Koči, A., Schirmer, W. \& Brunnacker, K. (1973): Paläomagnetische Daten aus dem mittleren Pleistozän des Rhein-Main-Raumes. - N. Jb. Geol. Paläont. Mh., Jg. 1973, H. 9: 545-554; Stuttgart.

Montrrans, H. M. van (1971): Palaeomagnetic Dating in the North Sea Basin. - Thesis: 133 S.; Rotterdam.

RAZI RAD, M. (1976): Schwermineraluntersuchungen zur Quartär-Stratigraphie am Mittelrhein. Sonderveröff. geol. Inst. Köln, 28: 164 S.; Köln.

Schönhals, E., Rohdenburg, H. \& Semmel, A. (1964): Ergebnisse neuerer Untersuchungen zur Würmlößgliederung in Hessen. - Eiszeitalter u. Gegenwart, 15: 199-206; OOhringen/Württ.

Semmel, A. (1967): Über Prä-Würm-Lösse in Hessen. - Notizbl. hess. L.-Amt Bodenforsch., 95: 239-241; Wiesbaden.

- (1968): Studien über den Verlauf jungpleistozäner Formung in Hessen. - Frankf. geogr. Hefte, 45: 133 S.; Frankfurt a. Main.

- (1969): Quartär. - In: Kümmerle, E. \& Semmel, A.: Erl. geol. Kt. Hessen $1: 25000$, Bl. 5916 Hochheim, 3. Aufl.: 51-99; Wiesbaden.

- (1973): Periglacial Sediments and their Stratigraphy. - In: State of Research on the Quaternary of the Federal Republic of Germany. - Eiszeitalter u. Gegenwart, 23/24: 293-305; Öhringen/Württ.

- (1974): Der Stand der Eiszeitforschung im Rhein-Main-Gebiet. - Rhein-Main. Forsch., 78: 9-56; Frankfurt a. Main.

- (1977): Grundzüge der Bodengeographie. - 120 S.; Stuttgart.

WIEGANK, F. (1975): Paläomagnetische Untersuchungen zur Quartärstratigraphie in der DDR (Mittelpleistozän von Voigtstedt). - Gerlands Beitr. Geophys., 84: 215-222; Leipzig.

Zagwijn, W. H., Montfrans, H. M. van \& Zandstra, J. G. (1971): Subdivision of the "Crome-

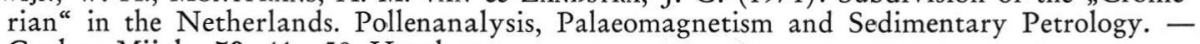
Geol. en Mijnb., 50: 41-58; Haarlem. 